\title{
Relative stochastic comparisons of additive frailty models
}

\author{
Mohamed Kayid ${ }^{1,2^{*}}$ (D) Salman Izadkhah ${ }^{3}$ and Mhamed Mesfioui ${ }^{4}$
}

${ }^{\text {"Correspondence: }}$

drkayid@ksu.edu.sa

1 Permanent address: Department of

Mathematics and Computer

Science, Faculty of Science, Suez

University, Suez, 41522, Egypt

${ }^{2}$ Department of Statistics and Operations Research, College of

Science, King Saud University,

Riyadh, Saudi Arabia

Full list of author information is

available at the end of the article

\begin{abstract}
This article is devoted to the proposal of two relative stochastic orders namely the relative hazard rate and relative mean residual life orders. These stochastic orders are applied to provide some stochastic comparisons between two additive frailty models. Some closure properties of the model with respect to these relative stochastic orders are presented. In addition, we demonstrate how the variation of the baseline variable and the variation of the additive variable in the additive frailty model, each in one time, has an effect on the model. Finally, a possible extension of the concept of relative orders to the multivariate case is discussed.
\end{abstract}

Keywords: stochastic order; hazard rate; mean residual life; additive hazards model; mixture; multivariate mean residual life; multivariate hazard rate

\section{Introduction and preliminaries}

In the context of reliability and survival analysis, various models have been introduced in the literature for modeling and analyzing failure time data. Some of these models are the proportional (additive) hazards model, the proportional (additive) reversed hazards model, and the proportional (additive) mean residual life model ( $c f$. [1-6]). The additive hazards model, which is well known in the literature, has played a prominent role in modeling survival data. For a non-negative random variable $X$ with the probability density function (pdf) $f$ and the survival function (sf) $\bar{F}$, the random variable $X_{x}=(X-x \mid X>x)$ for any $x \in \chi$ is known as the residual life of $X$ at some fixed time $x$ provided that $X$ is greater than $x$, where $\chi=\{x: \bar{F}(x)>0\}$. Denote by $r$ and $m$ the hazard rate (hr) and the mean residual life (mrl) functions of $X$, respectively, which are given by

$$
r(x)=\lim _{\delta \rightarrow 0^{+}} \frac{P\left(X_{x} \in(0, \delta]\right)}{\delta}=\frac{f(x)}{\bar{F}(x)}, \quad x \in \chi
$$

and

$$
m(x)=\frac{\int_{x}^{\infty} \bar{F}(u) d u}{\bar{F}(x)}, \quad x \in \chi
$$

which are connected by

$$
r(x)=\frac{1+\frac{d}{d x} m(x)}{m(x)}
$$

(c) 2016 Kayid et al. This article is distributed under the terms of the Creative Commons Attribution 4.0 International License (http://creativecommons.org/licenses/by/4.0/), which permits unrestricted use, distribution, and reproduction in any medium, provided you give appropriate credit to the original author(s) and the source, provide a link to the Creative Commons license, and indicate if changes were made. 
The additive hazards model considers a random variable $Z$, representing the changes in the operating conditions with an additive effect on $r(x)$ when $Z=z$, through the conditional hazard rate

$$
r(x \mid z)=r(x)+z, \quad x \geq 0
$$

Let $r(x \mid z)$ be the hr function of the conditional random variable $X(z)$. Then the sf of $X(z)$ is easily obtained:

$$
\bar{F}(x \mid z)=\bar{F}(x) e^{-x z}, \quad x \geq 0
$$

On the other hand, it has been recognized in many statistical procedures that individuals vary in their vulnerability to causes of death or disease, response to treatment, and impact of various risk factors. One way of taking this biological variation into account is through the observed covariates. However, it is not possible to incorporate all risk factors and all possible forms of inter-individual heterogeneity into consideration. In practice, the heterogeneity is an issue that cannot be explained by observed covariates, which is a serious problem because it may result in some misleading conclusions. To eliminate the problem of heterogeneity in many practical populations, researchers have considered the unconditional survival function of the additive hazards model (cf. [7-9]). As a result, the expression in (1) could be regarded as a consequence of the influence of a random environment on the hr $r(x)$. Formally, let $X(Z)$ be the overall random variable with the sf

$$
\bar{G}(x)=\bar{F}(x) E\left[e^{-x Z}\right], \quad x \geq 0,
$$

which is known as the additive frailty model (cf. [9]).

The aim of this paper is to propose two relative stochastic orders namely the relative mean residual life and relative hazard rate orders through which some relative stochastic comparisons between two additive frailty models are developed. The organization of this paper is as follows. In Section 2, main results of the paper are included. In Section 2.1, the definitions of the relative stochastic orders are given. Then we present some closure properties in Section 2.2. In Section 2.3, the variation of the baseline variable and in Section 2.4, the variation of the additive mixing effect on the additive frailty model are studied. A possible extension of the concept of relative stochastic orders to the multivariate case is discussed and some examples of interest are given in Section 2.5. Finally in Section 3, we close the paper with some remarks about the importance of the materials that will be discussed throughout.

Before proceeding further, we present some definitions and background for various stochastic comparisons ( $c f$. [10-12]). Throughout this paper $X_{1}$ and $X_{2}$ are two nonnegative and absolutely continuous random variables with pdf's $f_{1}$ and $f_{2}$, sf's $\bar{F}_{2}$ and $\bar{F}_{2}$, hr's $r_{1}$ and $r_{2}$ and mrl's $m_{1}$ and $m_{2}$, respectively. The term increasing is used instead of monotone non-decreasing and the term decreasing is used instead of monotone nonincreasing. We assume that all expectations are finite wherever they appear.

The random variable $X_{1}$ is said to be smaller than $X_{2}$ in the:

(i) Stochastic order (denoted $X_{1} \leq_{s t} X_{2}$ ) if $\bar{F}_{1}(x) \leq \bar{F}_{2}(x)$, for all $x \geq 0$.

(ii) Hazard rate order (denoted $X_{1} \leq_{h r} X_{2}$ ) if $\bar{F}_{2}(x) / \bar{F}_{1}(x)$ is increasing in $x \geq 0$. 
(iii) Likelihood ratio order (denoted $\left.X_{1} \leq{ }_{l r} X_{2}\right)$ if $f_{2}(x) / f_{1}(x)$ is increasing in $x \geq 0$.

(iv) Mean residual life order (denoted $X_{1} \leq_{m r} X_{2}$ ) if $\int_{x}^{\infty} \bar{F}_{2}(u) d u / \int_{x}^{\infty} \bar{F}_{1}(u) d u$ is increasing in $x \geq 0$.

(v) Aging intensity order (denoted $\left.X_{1} \leq_{A I} X_{2}\right)$ if $\int_{0}^{x}\left[r_{1}(u) / r_{1}(x)\right] d u \leq \int_{0}^{x}\left[r_{2}(u) / r_{2}(x)\right] d u$, for all $x \geq 0$.

The lifetime random variable $X_{1}$ is said to have:

(i) The increasing [decreasing] likelihood ratio (ILR, DLR) property if $f$ is a log-concave [log-convex] function on the support of $F_{1}$.

(ii) The increasing [decreasing] hazard rate (IFR, DFR) property if $\bar{F}$ is a log-concave [log-convex] function on the support of $F_{1}$.

(iii) Decreasing (increasing) mean residual life (DMRL, IMRL) property if $\int_{x}^{\infty} \bar{F}(u) d u$ is log-concave [log-convex] on the support of $F_{1}$.

The notion of total positivity (reverse regularity) is due to [13]. A non-negative measurable function $\beta(x, y)$ is said to be totally positive of order $2\left(\mathrm{TP}_{2}\right)$ [reverse regular of order 2 $\left.\left(\mathrm{RR}_{2}\right)\right]$ in $(x, y) \in \chi \times \gamma$, if

$$
\left|\begin{array}{ll}
\beta\left(x_{1}, y_{1}\right) & \beta\left(x_{1}, y_{2}\right) \\
\beta\left(x_{2}, y_{1}\right) & \beta\left(x_{2}, y_{2}\right)
\end{array}\right| \geq[\leq] 0
$$

for all $x_{1} \leq x_{2} \in \chi$ and $y_{1} \leq y_{2} \in \gamma$, in which $\chi$ and $\gamma$ are two real subsets of $\mathbb{R}$.

Finally, we will utilize frequently the following useful lemma from [14].

Lemma 1.1 Let $W \geq 0$ be a random variable with dffrom the family $\digamma=\left\{H_{\theta}, \theta \in \Theta \subseteq R\right\}$ such that $H_{\theta_{1}} \leq_{s t}\left(\geq_{s t}\right) H_{\theta_{2}}$, for all $\theta_{1} \leq \theta_{2} \in \Theta$. Suppose a measurable real function $\phi(w, \theta)$ for which $E_{\theta}[\phi(W, \theta)]$ exists. Then the function $E_{\theta}[\phi(W, \theta)]$ is:

(i) Decreasing in $\theta$, if $\phi(w, \theta)$ is decreasing (increasing) in $w$ and decreasing in $\theta$.

(ii) Increasing in $\theta$, if $\phi(w, \theta)$ is increasing (decreasing) in $w$ and increasing in $\theta$.

\section{Main results}

This section includes main results of the paper along with further discussions, conclusions and examples. Most of the stochastic orders discovered and analyzed in the literature are the ones which compare the 'location' or the 'magnitude' of the random variables and there are other ones which compare the 'variability' or the 'dispersion' of the random variables (cf. [11] and [12]). As another perspective of stochastic comparison between lifetime variables, we devoted our attention here to other kinds of stochastic orders which compare the random variables with respect to their 'aging' properties according to the well-known reliability measures of hazard rate and mean residual life. These stochastic orders called the relative hazard rate order and the relative mean residual life order, which will be discussed in the sequel, have been somewhat neglected in the literature. We provide further properties of these stochastic orders. Because there is no theoretical base for choosing the distribution of the additive effect variable in the additive frailty model it is important to see how the overall variable is influenced by the variation of the additive effect and baseline variables in the model. Such an investigation has been recently done in the context of various reliability models (see, for example, [15] and the references therein). Thus, as a second lead, we use the relative hazard and the relative mean residual life orders for comparison of additive hazards model. Possible extensions to the multivariate case are also discussed. 


\subsection{Relative orders}

In the context of reliability and survival analysis, a probabilistic order based on the monotonicity of the ratio $r_{1}(x) / r_{2}(x)$ is studied in $[16,17]$. The random variable $X_{1}$ is said to be aging faster than $X_{2}$ if $r_{1}(x) / r_{2}(x)$ is increasing in $x$. Based on this order relation, a generalized stochastic order has been introduced in [18]. The crossing hazards phenomenon has been studied in connection with prognostic studies in the treatment of breast cancer in [19]. An increasing hazards ratio is a reasonable alternative to the proportional hazards model in this case ( $c f$. [16]). According to the foregoing points we propose the following stochastic order.

Definition 2.1 The random variable $X_{1}$ is said to be smaller than $X_{2}$ in relative hazard rate order (denoted $\left.X_{1} \leq_{r l h r} X_{2}\right)$ if $r_{2}(x) / r_{1}(x)$ is decreasing in $x \geq 0$, or equivalently if $r_{i}(x)$ is $\mathrm{RR}_{2}$ in $(i, x) \in\{1,2\} \times[0, \infty)$.

It is to be noted here that the notion of the relative hazard rate order given in Definition 2.1 is equivalent to the notion of ageing faster given in [16] (see Definition 1 and Proposition 2.2 in [16]).

In replacement and repair strategies, along with the hr function, the mrl function also plays an important role as the latter summarizes the entire residual life function, whereas the former describes only the risk of instantaneous failure at some fixed time $x$. Therefore, in some situations, the mrl may be more appropriate than the hr in order to compare the lifetime of two devices. It is well known that $X_{2} \leq_{h r} X_{1}$ implies $X_{2} \leq_{m r} X_{1}$. To conclude the inverse implication, a sufficient condition is that the ratio $m_{2}(x) / m_{1}(x)$ is increasing in $x$ (cf. [11]). Therefore, the monotonicity property of the ratio of the mrl functions of two lifetime random variables seems to be important for analysis. Using the notion of relative $\mathrm{mrl}$, some useful properties in reliability theory were studied in [20,21].

Definition 2.2 The random variable $X_{1}$ is said to be smaller than $X_{2}$ in relative mrl order (denoted $X_{1} \leq_{r l m r} X_{2}$ ) if $m_{2}(x) / m_{1}(x)$ is increasing in $x \geq 0$, or equivalently if $m_{i}(x)$ is $\mathrm{TP}_{2}$ in $(i, x) \in\{1,2\} \times[0, \infty)$.

In the following result, we establish some useful implications regarding the relative hazard rate order and other well-known stochastic orders.

\section{Theorem 2.1}

(i) If $X_{1} \leq_{r l h r} X_{2}$ such that $X_{2}$ is IFR, then $X_{1}$ is IFR.

(ii) If $X_{1} \leq_{r l h r} X_{2}$ and $X_{2} \leq_{h r} X_{1}$, then $X_{2} \leq_{l r} X_{1}$.

(iii) $X_{1} \leq_{r l h r} X_{2}$ implies $X_{1} \leq_{A I} X_{2}$.

(iv) If $\lim _{x \rightarrow 0} \frac{f_{2}(x)}{f_{1}(x)} \leq 1$, then $X_{1} \leq_{r l h r} X_{2}$ implies $X_{1} \leq_{h r} X_{2}$.

(v) If $0<\lim _{x \rightarrow \infty} \frac{f_{2}(x)}{f_{1}(x)}<\infty$, then $X_{1} \leq_{r l h r} X_{2}$ implies $X_{2} \leq_{\text {lr }} X_{1}$.

Proof The proof of (i) is quite straightforward and hence omitted. The assertion (ii) has been given in Theorem 1.C.4(a) of [11]. To prove (iii), first observe that, because of $X_{1} \leq_{r l h r}$ $X_{2}$, we have

$$
\left[\frac{r_{2}(u)}{r_{1}(u)}-\frac{r_{2}(x)}{r_{1}(x)}\right] \geq 0, \quad \text { for all } u \leq x,
$$


which is equivalent to

$$
\left[\frac{r_{2}(u)}{r_{2}(x)}-\frac{r_{1}(u)}{r_{1}(x)}\right] \geq 0, \quad \text { for all } u \leq x
$$

Therefore

$$
\int_{0}^{x}\left[\frac{r_{2}(u)}{r_{2}(x)}-\frac{r_{1}(u)}{r_{1}(x)}\right] d u \geq 0
$$

which implies that $X_{1} \leq_{A I} X_{2}$. For proving (iv), note that $\bar{F}_{1}(0)=\bar{F}_{2}(0)=1$. Since $X_{1} \leq_{r l h r} X_{2}$ implies that $r_{2}(x) / r_{1}(x)$ is decreasing in $x>0$, we have

$$
\begin{aligned}
\frac{r_{2}(x)}{r_{1}(x)} & \leq \lim _{x \rightarrow 0}\left\{\frac{f_{2}(x)}{f_{1}(x)} \frac{\bar{F}_{1}(x)}{\bar{F}_{2}(x)}\right\} \\
& =\lim _{x \rightarrow 0} \frac{f_{2}(x)}{f_{1}(x)} \leq 1, \quad \text { for all } x>0,
\end{aligned}
$$

which obviously gives $X_{1} \leq_{h r} X_{2}$. For the sake of proving (v), observing that $\bar{F}_{1}(\infty)=$ $\bar{F}_{2}(\infty)=0$ and using the L'Hospital rule we get

$$
\begin{aligned}
\lim _{x \rightarrow \infty} \frac{\bar{F}_{1}(x)}{\bar{F}_{2}(x)} & =\lim _{x \rightarrow \infty} \frac{f_{1}(x)}{f_{2}(x)} \\
& =\left[\lim _{x \rightarrow \infty} \frac{f_{2}(x)}{f_{1}(x)}\right]^{-1} .
\end{aligned}
$$

Now, since $X_{1} \leq_{r l h r} X_{2}$ we have

$$
\begin{aligned}
\frac{r_{2}(x)}{r_{1}(x)} & \geq \lim _{x \rightarrow \infty} \frac{r_{2}(x)}{r_{1}(x)} \\
& =\lim _{x \rightarrow \infty} \frac{f_{2}(x)}{f_{1}(x)} \lim _{x \rightarrow \infty} \frac{\bar{F}_{1}(x)}{\bar{F}_{2}(x)}=1 .
\end{aligned}
$$

That is, $X_{2} \leq_{h r} X_{1}$, which in view of assertion (ii) implies that $X_{2} \leq_{l r} X_{1}$.

The following result illustrates another conclusion of the relative hazard rate order.

Theorem 2.2 Let $X_{1} \leq_{r l h r} X_{2}$ and $\lim _{x \rightarrow \infty}\left[r_{2}(x) / r_{1}(x)\right]=\alpha>0$. Then $\bar{F}_{2}(x) \leq \bar{F}_{1}^{\alpha}(x)$ for all $x>0$.

Proof Let $\kappa(x)=r_{2}(x) / r_{1}(x)$, since $X_{1} \leq_{r l h r} X_{2}$ we see that $\kappa(x)$ is non-negative and decreasing in $x \in(0, \infty)$. Because we have $\lim _{x \rightarrow \infty} \kappa(x)=\alpha>0$, we can get, for all $x>0$,

$$
\begin{aligned}
-\ln \left[\bar{F}_{2}(x)\right] & =\int_{0}^{x} \kappa(u) r_{1}(u) d u \\
& \geq \kappa(x) \int_{0}^{x} r_{1}(u) d u \\
& =-\kappa(x) \ln \left[\bar{F}_{1}(x)\right]
\end{aligned}
$$




$$
\begin{aligned}
& \geq-\ln \left[\bar{F}_{1}(x)\right] \lim _{x \rightarrow \infty} \kappa(x) \\
& =-\ln \left[\bar{F}_{1}(x)^{\alpha}\right] .
\end{aligned}
$$

Therefore, $\ln \left[\bar{F}_{2}(x)\right] \leq \ln \left[\bar{F}_{1}(x)^{\alpha}\right]$ for all $x>0$, which completes the proof.

The next result provides some characterization properties involving the relationship of the relative mean residual life order to other stochastic orders.

\section{Theorem 2.3}

(i) If $X_{1} \leq_{r l m r} X_{2}$ such that $X_{2}$ is DMRL, then $X_{1}$ is DMRL.

(ii) If $X_{1} \leq_{r l m r} X_{2}$ and $X_{2} \leq_{m r} X_{1}$, then $X_{2} \leq_{h r} X_{1}$.

(iii) If $0<\lim _{x \rightarrow \infty} \frac{f_{2}(x)}{f_{1}(x)}<\infty$, then $X_{1} \leq_{r l m r} X_{2}$ implies $X_{2} \leq_{h r} X_{1}$.

(iv) If $E\left(X_{1}\right) \leq E\left(X_{2}\right)$, then $X_{1} \leq_{r l m r} X_{2}$ implies $X_{1} \leq_{m r} X_{2}$.

Proof The proofs of (i) and (ii) are easily obtained and hence omitted. To prove (iii), by using the L'Hospital rule we have

$$
\begin{aligned}
\lim _{x \rightarrow \infty} \frac{\int_{x}^{\infty} \bar{F}_{2}(u) d u}{\int_{x}^{\infty} \bar{F}_{1}(u) d x} & =\lim _{x \rightarrow \infty} \frac{\bar{F}_{2}(x)}{\bar{F}_{1}(x)} \\
& =\lim _{x \rightarrow \infty} \frac{f_{2}(x)}{f_{1}(x)}
\end{aligned}
$$

Now, let us observe that

$$
\begin{aligned}
\frac{m_{2}(x)}{m_{1}(x)} & \leq \lim _{x \rightarrow \infty} \frac{m_{2}(x)}{m_{1}(x)} \\
& =\lim _{x \rightarrow \infty} \frac{\int_{x}^{\infty} \bar{F}_{2}(u) d u}{\int_{x}^{\infty} \bar{F}_{1}(u) d u} \lim _{x \rightarrow \infty} \frac{\bar{F}_{1}(x)}{\bar{F}_{2}(x)} \\
& =\lim _{x \rightarrow \infty} \frac{f_{2}(x)}{f_{1}(x)}\left[\lim _{x \rightarrow \infty} \frac{f_{2}(x)}{f_{1}(x)}\right]^{-1}=1, \quad \text { for all } x \geq 0 .
\end{aligned}
$$

Therefore, we conclude that $X_{2} \leq_{m r} X_{1}$, which by the result of assertion (ii) implies that $X_{2} \leq_{h r} X_{1}$. To prove (iv), for all $x \geq 0$ we have

$$
\frac{m_{2}(x)}{m_{1}(x)} \geq \frac{m_{2}(0)}{m_{1}(0)}=\frac{E\left[X_{2}\right]}{E\left[X_{1}\right]} \geq 1
$$

and the result is now deduced.

In view of the fact that the hr order implies the mrl order (cf. [22]), one may wonder whether the relative hazard rate order implies the relative mean residual life order. Example 2.1 below gives a negative answer.

Example 2.1 Let $X_{1}$ and $X_{2}$ have respective survival functions

$$
\bar{F}_{1}(x)= \begin{cases}\exp (-x), & 0 \leq x \leq 1, \\ x \exp (-x), & x \geq 1,\end{cases}
$$


and

$$
\bar{F}_{2}(x)= \begin{cases}\exp (-2 x), & 0 \leq x \leq 1 \\ x^{2} \exp (-2 x), & x \geq 1\end{cases}
$$

Obviously, $\bar{F}_{2}(x)=\left[\bar{F}_{1}(x)\right]^{2}$, for all $x \in \mathbb{R}$, which means $X_{1}$ and $X_{2}$ have proportional hazards rate and hence $X_{1} \leq_{r l h r} X_{2}$. After straightforward computation, one obtains

$$
\frac{m_{2}(x)}{m_{1}(x)}= \begin{cases}\frac{4+6 \exp (2(x-1))}{8(1+\exp (x-2))}, & 0 \leq x \leq 1, \\ \frac{2 x^{2}+2 x+1}{4 x(x+1)}, & x \geq 1\end{cases}
$$

The ratio $m_{2}(x) / m_{1}(x)$ does not have a monotone behavior and hence neither $X_{1} \leq_{r l m r} X_{2}$ nor $X_{2} \leq_{r l m r} X_{1}$ holds.

In general it seems that there is no relation between the relative hazard rate and the relative mean residual life orders. In spite of this, the following result gives a sufficient condition under which each of these orders implies the other one. The proof is straightforward and hence we omit it.

Theorem 2.4 Let $\frac{1+\frac{d}{d x} m_{1}(x)}{1+\frac{d}{d x} m_{2}(x)}$ be increasing (decreasing) in $x$ over $[0, \infty)$. Then

$$
X_{1} \leq_{r l m r} X_{2} \quad \Rightarrow \quad X_{1} \leq_{r l h r} X_{2} \quad\left(X_{1} \leq_{r l h r} X_{2} \quad \Rightarrow \quad X_{1} \leq_{r l m r} X_{2}\right)
$$

\subsection{Closure properties of the model}

Let $\lambda$ be the hazard rate function of $X(Z)$. Below we state the closure property of the additive frailty model with respect to the relative hazard rate order when appropriate assumptions are satisfied.

Theorem $2.5 X(Z) \leq_{r l h r} X$ provided that $X$ is IFR.

Proof In view of Theorem 2.1 in [9], $\lambda(x)-r(x)=E[Z \mid X(Z)>x]$, which is decreasing in $x$ from Corollary 2.1 in [9]. Since $X$ is IFR by assumption, the result follows.

To state and prove another closure property of the additive frailty model with respect to the relative mean residual life order, we recall the following lemma from [14].

Lemma 2.1 Let $f(x, y)$ be an $R R_{2}\left(T P_{2}\right)$ function in $(x, y) \in[0, \infty) \times[0, \infty)$, and let $\phi_{i}(y)$ be $T P_{2}$ in $(i, y) \in\{1,2\} \times[0, \infty)$. Then

$$
\Phi_{i}(x)=\int_{0}^{\infty} \phi_{i}(y) f(x, y) d y
$$

is $R R_{2}\left(T P_{2}\right)$ in $(i, x) \in\{1,2\} \times[0, \infty)$.

Theorem 2.6 $X \leq_{r l m r} X(Z)$ provided that $X$ is IFR. 
Proof We need to show that $l(x) / m(x)$ is increasing in $x \geq 0$, where $l$ is the mrl function of $X(Z)$. By (3), we get

$$
\begin{aligned}
\frac{l(x)}{m(x)} & =\frac{\bar{F}(x) \int_{x}^{\infty} \bar{G}(u) d u}{\bar{G}(x) \int_{x}^{\infty} \bar{F}(u) d u} \\
& =\frac{E\left[\int_{x}^{\infty} \bar{F}(u) e^{-u Z} d u\right]}{E\left[\int_{x}^{\infty} \bar{F}(u) e^{-x Z} d u\right]} \\
& =\int_{0}^{\infty}\left[\frac{\int_{x}^{\infty} \bar{F}(u) e^{-u w} d u}{e^{-x w} \int_{x}^{\infty} \bar{F}(u) d u}\right] \frac{e^{-x w} h(w)}{\int_{0}^{\infty} e^{-x w} h(w) d w} d w \\
& =E\left[\phi\left(W_{x}, x\right)\right],
\end{aligned}
$$

where $W_{x}$ is a non-negative random variable with pdf

$$
h(w ; x)=\frac{e^{-x w} h(w)}{\int_{0}^{\infty} e^{-x w} h(w) d w}, \quad w \geq 0,
$$

and

$$
\begin{aligned}
\phi(w, x) & =\frac{\int_{x}^{\infty} \bar{F}(u) e^{-u w} d u}{e^{-x w} \int_{x}^{\infty} \bar{F}(u) d u} \\
& =\frac{\int_{0}^{\infty} \bar{F}(x+y) e^{-w y} d y}{\int_{0}^{\infty} \bar{F}(x+y) d y} .
\end{aligned}
$$

It can be shown that $W_{x}$ is stochastically decreasing in $x$, and $\phi(w, x)$ is decreasing in $w$. For each $w \geq 0$, let

$$
\phi_{i}(y)= \begin{cases}1, & i=2, \\ e^{-w y}, & i=1,\end{cases}
$$

and take $f(x, y)=\bar{F}(x+y)$ in Lemma 2.1. We observe that $\phi_{i}(y)$ is $\mathrm{TP}_{2}$ in $(i, y) \in\{1,2\} \times$ $[0, \infty)$. By the IFR property of $X$, we see that $f(x, y)$ is $\mathrm{RR}_{2}$ in $(x, y) \in[0, \infty) \times[0, \infty)$. As a result of Lemma 2.1, for each fixed $w \geq 0, \Phi_{i}(x)$ is $\mathrm{RR}_{2}$ in $(i, x) \in\{1,2\} \times[0, \infty)$, which means that $\Phi_{2}(x) / \Phi_{1}(x)$ in decreasing in $x>0$, that is, $\phi(w, x)=\Phi_{1}(x) / \Phi_{2}(x)$ is increasing in $x>0$, for all $w \geq 0$. Now, Lemma 1.1(ii) completes the proof.

\subsection{Variation in mixing variable}

Let $Z_{i}$ be a mixing effect having pdf $h_{i}$ and let $X\left(Z_{i}\right)$ be the corresponding overall variable in the additive frailty model with the sf

$$
\bar{G}_{i}(x)=\bar{F}(x) E\left[e^{-x Z_{i}}\right], \quad x \geq 0, i=1,2 .
$$

We will assume, for each $i=1,2$, that $X\left(Z_{i}\right)$ has pdf $g_{i}$ given by

$$
g_{i}(x)=E\left[e^{-x Z_{i}}\left(f(x)+Z_{i} \bar{F}(x)\right)\right], \quad x \geq 0,
$$


and we assume that $\lambda_{i}$ is the hr function of $X\left(Z_{i}\right)$ obtained from (4) and (5) by

$$
\lambda_{i}(x)=\frac{E\left[e^{-x Z_{i}}\left(r(x)+Z_{i}\right)\right]}{E\left[e^{-x Z_{i}}\right]}, \quad x \geq 0
$$

In the following result we discuss conditions on the two additive effects $Z_{1}$ and $Z_{2}$ under which the resulted overall variables from this effects with sf as in (4) are ordered according to the relative hazard rate order. We assume that $Z_{1}$ and $Z_{2}$ are statistically independent, $Z=Z_{1}+Z_{2}$ and $e_{i}(z)=E\left(Z_{i} \mid Z=z\right), i=1,2$.

Theorem 2.7 Let $X$ be IFR and let $e_{1}(z)$ be decreasing in $z \geq 0$. Then $X\left(Z_{1}\right) \leq$ rlhr $X\left(Z_{2}\right)$.

Proof We need to demonstrate that $\lambda_{1}(x) / \lambda_{2}(x)$ is increasing in $x>0$. Suppose that $h_{Z}$ is the pdf of $Z=Z_{1}+Z_{2}$. Note that by the assumption because of the identity $e_{2}(z)+e_{1}(z)=z$, for any $z \in[0, \infty), e_{2}(z)$ is increasing in $z$. In view of (6) and because $Z_{1}$ and $Z_{2}$ are independent one can get, for all $x>0$,

$$
\begin{aligned}
\frac{\lambda_{1}(x)}{\lambda_{2}(x)} & =\frac{E\left[e^{-x Z_{1}}\left(r(x)+Z_{1}\right)\right] E\left[e^{-x Z_{2}}\right]}{E\left[e^{-x Z_{2}}\left(r(x)+Z_{2}\right)\right] E\left[e^{\left.-x Z_{1}\right]}\right.} \\
& =\frac{E\left[e^{-x\left(Z_{1}+Z_{2}\right)}\left(r(x)+Z_{1}\right)\right]}{E\left[e^{-x\left(Z_{1}+Z_{2}\right)}\left(r(x)+Z_{2}\right)\right]} \\
& =\frac{E\left[e^{-x Z}\left\{r(x)+e_{1}(Z)\right\}\right]}{E\left(e^{-x Z}\left\{r(x)+e_{2}(Z)\right\}\right)} \\
& =\int_{0}^{\infty}\left[\frac{r(x)+e_{1}(w)}{r(x)+e_{2}(w)}\right] \frac{\left\{r(x)+e_{2}(w)\right\} e^{-x w} h_{Z}(w)}{\int_{0}^{\infty}\left\{r(x)+e_{2}(w)\right\} e^{-x w} h_{Z}(w) d w} d w \\
& =E[\phi(W, x)],
\end{aligned}
$$

where

$$
\phi(w, x)=\frac{r(x)+e_{1}(w)}{r(x)+e_{2}(w)}, \quad w, x \geq 0,
$$

and $W$ is a non-negative rv with pdf

$$
h(w \mid x)=\frac{\left[r(x)+e_{2}(w)\right] e^{-x w} h_{Z}(w)}{\int_{0}^{\infty}\left[r(x)+e_{2}(w)\right] e^{-x w} h_{Z}(w) d w}, \quad w, x \geq 0 .
$$

From the assumptions we see that $\phi$ is decreasing in $w \geq 0$. Set $e(w)=e_{1}(w)-e_{2}(w)$ and note that $e_{1}(0)=e_{2}(0)=0$, by which we have $e(0)=0$. Because $e_{1}$ is decreasing and $e_{2}$ is increasing, $e$ is decreasing and hence $e(w) \leq e(0)=0$, for all $w \geq 0$, i.e. $e$ is a non-positive function. Therefore, we see that

$$
\phi(w, x)=1+\frac{e(w)}{r(x)+e_{2}(w)}
$$

is increasing in $x>0$ since $r$ (the hr function of $X$ ) is increasing by the assumption. On the other hand, it is easy to show that $h(w \mid x)$ is $\mathrm{RR}_{2}$ in $(w, x) \in[0, \infty) \times[0, \infty)$, which implies that $W$ is stochastically decreasing in $x$. By applying Lemma 1.1(ii), the proof is completed. 
The next result facilitates the use of one of the conditions in Theorem 2.7.

Proposition 2.1 If $Z_{2}$ is DLR, then $e_{1}(z)$ is decreasing in $z \in[0, \infty)$.

Proof Denote by $h_{Z}$ the pdf of $Z=Z_{1}+Z_{2}$ and denote by $h_{i}$ the pdf of $Z_{i}, i=1,2$. If we denote by $h$ the joint pdf of $\left(Z_{1}, Z_{2}\right)$ then by the fact that $Z_{1}$ and $Z_{2}$ are independent, we have $h\left(z_{1}, z_{2}\right)=h_{1}\left(z_{1}\right) h_{2}\left(z_{2}\right)$, for any $\left(z_{1}, z_{2}\right) \in[0, \infty) \times[0, \infty)$. Therefore,

$$
\begin{aligned}
e_{1}(z) & =E\left[Z_{1} \mid Z_{1}+Z_{2}=z\right] \\
& =\int_{0}^{\infty} z_{1} h_{Z_{1} \mid Z=z}\left(z_{1} \mid z\right) d z_{1} \\
& =\frac{\int_{0}^{\infty} z_{1} h_{1}\left(z_{1}\right) h_{2}\left(z-z_{1}\right) d z_{1}}{\int_{0}^{\infty} h_{1}\left(z_{1}\right) h_{2}\left(z-z_{1}\right) d z_{1}}, \quad \text { for any } z \geq 0 .
\end{aligned}
$$

Now, it is adequate to see that $\rho(i, z)=\int_{0}^{\infty} \phi\left(i, z_{1}\right) \psi\left(z_{1}, z\right) d z_{1}$ is $\mathrm{RR}_{2}$ as a function of $i=1,2$ and of $z \in[0, \infty)$, where $\phi\left(1, z_{1}\right)=1$ and $\phi\left(2, z_{1}\right)=z_{1}$, and $\psi\left(z_{1}, z\right)=h_{1}\left(z_{1}\right) h_{2}\left(z-z_{1}\right)$. It is easy to see that $\phi\left(i, z_{1}\right)$ is $\mathrm{TP}_{2}$ in $\left(i, z_{1}\right) \in\{1,2\} \times[0, \infty)$, and also because $Z_{2}$ is DLR; thus its pdf is log-convex which shows that $\psi\left(z_{1}, z\right)$ is $\mathrm{RR}_{2}$ in $\left(z_{1}, z\right) \in[0, \infty) \times[0, \infty)$. An application of the basic composition formula, as reported in Lemma 2.1, will complete the proof.

Example 2.2 Suppose that $Z_{1}$ and $Z_{2}$ have exponential distributions with means $1 / \theta_{1}$ and $1 / \theta_{2}$, respectively, where $\theta_{1}>\theta_{2}$. Suppose also that the baseline variable $X$ follows an exponential distribution with mean $1 / \theta$. It is evident that $Z_{2}$ is DLR and that $X$ is IFR. By Proposition 2.1, $e_{1}(z)$ is decreasing in $z$. Hence, the assumptions of Theorem 2.7 hold true. After some routine calculations, one has $\lambda_{i}(x)=1 /\left(x+\theta_{i}\right)+\theta, i=1$, 2 . It can be seen that $\lambda_{2}$ decreases faster than $\lambda_{1}$ so that $\lambda_{1} / \lambda_{2}$ is increasing, i.e., $X\left(Z_{1}\right) \leq_{r l h r} X\left(Z_{2}\right)$, which confirms the result of Theorem 2.7 .

In the rest of this subsection we study the problem of comparison of two additive frailty models in (4) based on the relative mean residual life order. Note that, in view of (2), the $\mathrm{mrl}$ function of $X(z)$ is given by

$$
m(x \mid z)=\int_{x}^{\infty} \frac{\bar{F}(u) e^{-u z}}{\bar{F}(x) e^{-x z}} d u, \quad x, z \geq 0
$$

Let $l_{i}(x)$ denote the mrl function of $X\left(Z_{i}\right)$ with the sf of (4). By some calculation we have, for each $i=1,2$,

$$
\begin{aligned}
l_{i}(x) & =E\left[X\left(Z_{i}\right)-x \mid X\left(Z_{i}\right)>x\right] \\
& =\int_{x}^{\infty} \frac{\bar{F}(u) E\left[e^{-u Z_{i}}\right]}{\bar{F}(x) E\left[e^{-x Z_{i}}\right]} d u \\
& =\frac{E\left[\int_{x}^{\infty} \bar{F}(u) e^{-u Z_{i}} d u\right]}{E\left[\bar{F}(x) e^{-x Z_{i}}\right]} .
\end{aligned}
$$

Now, the following result is immediate. 
Theorem 2.8 Let $Z_{1} \leq_{l r} Z_{2}$ such that $Z_{2}$ is DLR and let

$$
X\left(z_{1}\right) \leq_{\text {rlmr }} X\left(z_{2}\right), \quad \text { for all } z_{1} \leq z_{2} \in[0, \infty) .
$$

Then

$$
X\left(Z_{1}\right) \leq_{r l m r} X\left(Z_{2}\right) .
$$

Proof Because of (8) and because $Z_{1}$ and $Z_{2}$ are independent, we can get

$$
\begin{aligned}
\frac{l_{2}(x)}{l_{1}(x)} & =\frac{\left.E e^{-x Z_{2}} m\left(x \mid Z_{2}\right)\right] E\left[e^{-x Z_{1}}\right]}{E\left[e^{-x Z_{1}} m\left(x \mid Z_{1}\right)\right] E\left[e^{-x Z_{2}}\right]} \\
& =\frac{E\left[e^{-x\left(Z_{1}+Z_{2}\right)} m\left(x \mid Z_{2}\right)\right]}{E\left[e^{-x\left(Z_{1}+Z_{2}\right)} m\left(x \mid Z_{1}\right)\right]}, \quad \text { for all } x \geq 0 .
\end{aligned}
$$

It therefore suffices to prove that

$$
\rho(i, x)=E\left[e^{-x\left(Z_{1}+Z_{2}\right)} m\left(x \mid Z_{i}\right)\right]
$$

is $\operatorname{TP}_{2}$ in $(i, x) \in\{1,2\} \times[0, \infty)$. Before indicating that, by conditioning on $Z=Z_{1}+Z_{2}$, we have

$$
\begin{aligned}
\rho(i, x) & =E\left[e^{-x\left(Z_{1}+Z_{2}\right)} m\left(x \mid Z_{i}\right)\right] \\
& =E\left\{E\left[e^{-x\left(Z_{1}+Z_{2}\right)} m\left(x \mid Z_{i}\right) \mid Z\right]\right\} \\
& =E\left\{e^{-x Z} E\left[m\left(x \mid Z_{i}\right) \mid Z\right]\right\}, \quad i=1,2 .
\end{aligned}
$$

Now, it is enough to prove that $\rho(2, x) / \rho(1, x)$ is increasing in $x \geq 0$. First of all, suppose that $f_{Z}$ denotes the pdf of $Z=Z_{1}+Z_{2}$. Then

$$
\begin{aligned}
\frac{\rho(2, x)}{\rho(1, x)} & =\frac{\int_{0}^{\infty} e^{-x w} E\left[m\left(x \mid Z_{2}\right) \mid Z=w\right] f_{Z}(w) d w}{\int_{0}^{\infty} e^{-x w} E\left[m\left(x \mid Z_{1}\right) \mid Z=w\right] f_{Z}(w) d w} \\
& =E\left[\phi\left(W_{x}, x\right)\right],
\end{aligned}
$$

where $\phi(w, x)=E\left[m\left(x \mid Z_{2}\right) \mid Z=w\right] / E\left[m\left(x \mid Z_{1}\right) \mid Z=w\right], w \geq 0$, and $W_{x}$ is a non-negative random variable with $\mathrm{pdf}$

$$
h(w \mid x)=\frac{e^{-x w} f_{Z}(w) E\left[m\left(x \mid Z_{1}\right) \mid Z=w\right]}{\int_{0}^{\infty} e^{-x w} f_{Z}(w) E\left[m\left(x \mid Z_{1}\right) \mid Z=w\right] d w}, \quad w \geq 0 .
$$

Now, we can write

$$
\phi(w, x)=\frac{\int_{0}^{w} m(x \mid y) f_{Z_{2} \mid Z=w}(y) d y}{\int_{0}^{w} m(x \mid y) f_{Z_{1} \mid Z=w}(y) d y} .
$$

Define for each fixed $w \geq 0$

$$
\Psi(i, x)=\int_{0}^{w} m(x \mid y) f_{Z_{i} \mid Z=w}(y) d y, \quad i=1,2 .
$$


The purpose is now to prove that:

1. $\phi(w, x)$ is increasing in $x$, for all $w \geq 0$.

2. $\phi(w, x)$ is decreasing in $w$, for all $x>0$.

3. $W_{x}$ is stochastically decreasing in $x$.

Denote by $h\left(z_{1}, z_{2}\right)$ the values of the joint pdf of $\left(Z_{1}, Z_{2}\right)$. From (9) we observe that $m(x \mid y)$ is $\mathrm{TP}_{2}$ in $(x, y)$, and

$$
\begin{aligned}
\frac{f_{Z_{2} \mid Z=w}(y)}{f_{Z_{1} \mid Z=w}(y)} & =\frac{h(w-y, y)}{h(y, w-y)} \\
& =\frac{h_{2}(y)}{h_{1}(y)} \frac{h_{1}(w-y)}{h_{2}(w-y)}
\end{aligned}
$$

is increasing in $y$, i.e. $f_{Z_{i} \mid Z=w}(y)$ is $\mathrm{TP}_{2}$ in $(i, y)$. Lemma 2.1 shows that $\Psi(i, x)$ is $\mathrm{TP}_{2}$ in $(i, x) \in$ $\{1,2\} \times[0, \infty)$, and hence $\phi(w, x)$ is increasing in $x$. On the other hand, the identity of $\phi(w, x)=E\left[\phi_{0}(Y, w)\right]$ holds for all $w \geq 0$, in which

$$
\begin{aligned}
\phi_{0}(y, w) & =\frac{f_{Z_{2} \mid Z=w}(y)}{f_{Z_{1} \mid Z=w}(y)} \\
& =\frac{h_{2}(y) h_{1}(w-y)}{h_{1}(y) h_{2}(w-y)}, \quad y \in(0, w],
\end{aligned}
$$

which is decreasing in $w \geq 0$ and increasing in $y$, because $Z_{1} \leq_{l r} Z_{2}$. For each fixed $x>0$, $Y$ is a non-negative random variable with $\mathrm{pdf}$

$$
\begin{aligned}
h_{0}(y \mid w) & =\frac{m(x \mid y) f_{Z_{1} \mid Z=w}(y)}{\int_{0}^{w} m(x \mid y) f_{Z_{1} \mid Z=w}(y) d y} \\
& =\frac{m(x \mid y)}{C(w)} f_{Z_{1} \mid Z=w}(y), \quad y \in(0, w],
\end{aligned}
$$

where $C(w)$ is the normalizing constant. For all $w_{1} \leq w_{2} \in[0, \infty)$, we have

$$
\begin{aligned}
\frac{h_{0}\left(y \mid w_{2}\right)}{h_{0}\left(y \mid w_{1}\right)} & =\frac{C\left(w_{1}\right)}{C\left(w_{2}\right)} \frac{f_{Z_{1} \mid Z=w_{2}}(y)}{f_{Z_{1} \mid Z=w_{1}}(y)} \\
& =\frac{C\left(w_{1}\right)}{C\left(w_{2}\right)} \frac{h_{2}\left(w_{2}-y\right)}{h_{2}\left(w_{1}-y\right)} .
\end{aligned}
$$

We know that $h_{2}$ is log-convex, thus $h_{0}\left(y \mid w_{2}\right) / h_{0}\left(y \mid w_{1}\right)$ is decreasing in $y$ and therefore $Y$ is stochastically decreasing in $w$. Lemma 1.1(i) implies that $\phi(w, x)$ is decreasing in $w \geq 0$. We have, for all $w_{1} \leq w_{2} \in[0, \infty)$,

$$
\frac{h\left(w_{2} \mid x\right)}{h\left(w_{1} \mid x\right)} \propto[\bar{G}(x)]^{w_{2}-w_{1}} \frac{E\left\{m\left(x \mid Z_{1}\right) \mid Z=w_{2}\right\}}{E\left\{m\left(x \mid Z_{1}\right) \mid Z=w_{1}\right\}} .
$$

We can establish that

$$
\frac{E\left[m\left(x \mid Z_{1}\right) \mid Z=w_{2}\right]}{E\left[m\left(x \mid Z_{1}\right) \mid Z=w_{1}\right]}=\frac{\int_{0}^{\infty} m(x \mid y) f_{Z_{1} \mid Z=w_{2}}(y) d y}{\int_{0}^{\infty} m(x \mid y) f_{Z_{1} \mid Z=w_{1}}(y) d y}
$$


We can verify that $f_{Z_{1} \mid Z=w_{i}}(y)$ is $\mathrm{RR}_{2}$ in $(i, y) \in\{1,2\} \times[0, \infty)$, and we, moreover, know that $m(x \mid y)$ is $T P_{2}$ in $(x, y) \in[0, \infty) \times[0, \infty)$. Hence, applying Lemma 2.1,

$$
\Psi^{*}(i, x)=\int_{0}^{\infty} m(x \mid y) f_{Z_{1} \mid Z=w_{i}}(y) d y
$$

is $\mathrm{RR}_{2}$ in $(i, x) \in\{1,2\} \times[0, \infty)$. This means that $W_{x}$ is stochastically decreasing in $x$. Repeated application of Lemma 1.1(i) to the identity given in (10) shows that $E\left[\phi\left(W_{x}, x\right)\right]$ is increasing in $x$, and hence the proof is completed.

The next example indicates that the condition that $Z_{2}$ is DLR in Theorem 2.8 is not a necessary one.

Example 2.3 Suppose $Z_{i}$ has density $h_{i}(z)=\theta_{i}^{2}(z+\theta) e^{-z \theta_{i}} /\left(1+\theta_{i} \theta\right), i=1,2$ in which $\theta_{1}>\theta_{2}$. It can easily be shown that $Z_{1} \leq_{l r} Z_{2}$ and that $Z_{2}$ is not DLR. If $X$ is an exponential random variable with mean $1 / \theta$, then $m(x \mid z)=1 /(\theta+z)$. Thus, $X\left(z_{1}\right) \leq_{r l m r} X\left(z_{2}\right)$, for all $z_{1} \leq z_{2}$. Hence, the second assumption in Theorem 2.8 is not satisfied. It can be checked, after calculations, that $l_{i}(x)=1 /\left(x+\theta_{i}\right)+\theta, i=1,2$. Now, one gets $X\left(Z_{1}\right) \leq{ }_{r l m r} X\left(Z_{2}\right)$, which says that the result of Theorem 2.8 remains true.

\subsection{Variation in baseline variable}

In this subsection, we discuss the impact of different baseline variables sharing the same additive effect into the additive frailty model. Let $X_{i}$ be the baseline variable with the sf $\bar{F}_{i}$, the pdf $f_{i}$ and hr function $r_{i}, i=1,2$. Let $X_{i}(Z)$ be the overall variable arising from the baseline variable $X_{i}$ with additive mixing effect $Z$ and denote its sf by $\bar{F}_{i}^{*}$, which is given by

$$
\bar{F}_{i}^{*}(x)=\bar{F}_{i}(x) E\left[e^{-x Z}\right], \quad x \geq 0, i=1,2 .
$$

We use $\lambda_{i}^{*}$ to denote the $\operatorname{hrf}$ of $X_{i}(Z)$, for each $i=1,2$, which is given by

$$
\lambda_{i}^{*}(x)=\frac{E\left[e^{-x Z}\left(r_{i}(x)+Z\right)\right]}{E\left[e^{-x Z}\right]}, \quad x \geq 0 .
$$

Below we make the rhr order between $X_{1}(Z)$ and $X_{2}(Z)$ under some appropriate assumptions.

Theorem 2.9 Let $X_{1}$ be IFR and $X_{2}$ be DFR such that $f_{2}\left(0^{+}\right) \leq f_{1}\left(0^{+}\right)$, where $f_{i}\left(0^{+}\right)=$ $\lim _{x \rightarrow 0^{+}} f_{i}(x)$ for each $i=1,2$. Then $X_{1}(Z) \leq r l h r$

Proof We need to establish that $\lambda_{2}^{*}(x) / \lambda_{1}^{*}(x)$ is decreasing in $x>0$. To this end, from (12) we can get

$$
\begin{aligned}
\frac{\lambda_{2}^{*}(x)}{\lambda_{1}^{*}(x)} & =\frac{E\left[e^{-x Z}\left(Z+r_{2}(x)\right)\right]}{E\left[e^{-x Z}\left(Z+r_{1}(x)\right)\right]} \\
& =\int_{0}^{\infty}\left[\frac{w+r_{2}(x)}{w+r_{1}(x)}\right] \frac{\left[w+r_{1}(x)\right] e^{-x w} h(w)}{\int_{0}^{\infty}\left[w+r_{1}(x)\right] e^{-x w} h(w) d w} d w \\
& =E[\phi(W, x)],
\end{aligned}
$$


where $\phi(w, x)=\left[w+r_{2}(x)\right) /\left(w+r_{1}(x)\right]$, which by the assumptions is decreasing in $x>0$, for each fixed $w \geq 0$, and, moreover, $W$ is a non-negative rv with pdf

$$
h(w \mid x)=\frac{\left[w+r_{1}(x)\right] e^{-x w} h(w)}{\int_{0}^{\infty}\left[w+r_{1}(x)\right] e^{-x w} h(w) d w}, \quad w \geq 0 .
$$

Note that we can write $\phi(w, x)=1-\delta(x) /\left(w+r_{1}(x)\right)$, with $\delta(x)=r_{1}(x)-r_{2}(x)$. By the assumptions, $\delta$ is increasing and thus

$$
\begin{aligned}
\delta(x) & =r_{1}(x)-r_{2}(x) \\
& \geq \lim _{x \rightarrow 0}\left[r_{1}(x)-r_{2}(x)\right] \\
& =f_{1}\left(0^{+}\right)-f_{2}\left(0^{+}\right) \geq 0, \quad \text { for all } x>0 .
\end{aligned}
$$

Because of these observations, we can easily see that $\phi$ is increasing in $w \geq 0$, for each fixed $x>0$. One can easily show that $h(w \mid x)$ is $\mathrm{RR}_{2}$ in $(w, x)$, which implies that $W$ is stochastically decreasing in $x$. By Lemma 1.1(i) we obtain the proof.

Example 2.4 Consider two baseline variables $X_{1}$ and $X_{2}$ with pdf's $f_{1}(x)=(x+1) e^{-x-x^{2} / 2}$ and $f_{2}(x)=e^{-x / 2} / 2$, respectively. It is seen that $r_{1}(x)=x+1$ and that $r_{2}(x)=1 / 2$. Thus, $X_{1}$ is IFR and $X_{2}$ is DFR. It is also observed that $f_{2}\left(0^{+}\right)=1 / 2<1=f_{1}\left(0^{+}\right)$. Based on Theorem 2.9, we must have $X_{1}(Z) \leq_{r l h r} X_{2}(Z)$ for any random effect $Z$. Let $Z$ have an exponential distribution with mean $1 / \theta$. It can then be directly derived that $\lambda_{1}^{*}(x)=1 /(x+\theta)+x+1$ and that $\lambda_{2}^{*}(x)=1 /(x+\theta)+1 / 2$. Hence, $\lambda_{1}^{*} / \lambda_{2}^{*}$ is an increasing function which validates the result of Theorem 2.9 as expected.

Now, assume that $X_{i}(Z)$ with the $\sin (11)$ has the $\operatorname{mrl}$ function $l_{i}^{*}, i=1,2$. In the following, the relative mean residual life order between $X_{1}(Z)$ and $X_{2}(Z)$ holds when appropriate assumptions are imposed.

Theorem 2.10 Let $X_{1} \leq_{h r} X_{2}$ and let

$$
X_{1}(z) \leq_{\text {rlmr }} X_{2}(z), \quad \text { for all } z \geq 0 \text {. }
$$

Then

$$
X_{1}(Z) \leq_{r l m r} X_{2}(Z)
$$

Proof The proof will be obtained if we show that $l_{2}^{*}(x) / l_{1}^{*}(x)$ is increasing in $x \geq 0$. We have

$$
\begin{aligned}
\frac{l_{2}^{*}(x)}{l_{1}^{*}(x)} & =\frac{E\left[\bar{F}_{1}(x) \int_{x}^{\infty} \bar{F}_{2}(u) e^{-u Z} d u\right]}{E\left[\bar{F}_{2}(x) \int_{x}^{\infty} \bar{F}_{1}(u) e^{-u Z} d u\right]} \\
& =\int_{0}^{\infty}\left[\frac{\bar{F}_{1}(x) \int_{x}^{\infty} \bar{F}_{2}(u) e^{-u w} d u}{\bar{F}_{2}(x) \int_{x}^{\infty} \bar{F}_{1}(u) e^{-u w} d u}\right] \frac{\left[\int_{x}^{\infty} \bar{F}_{1}(u) e^{-u w} d u\right] h(w)}{\int_{0}^{\infty}\left[\int_{x}^{\infty} \bar{F}_{1}(u) e^{-u w} d u\right] h(w) d w} d w \\
& =E[\phi(W, x)],
\end{aligned}
$$


where

$$
\begin{aligned}
\phi(w, x) & =\frac{\bar{F}_{1}(x) \int_{x}^{\infty} \bar{F}_{2}(u) e^{-u w} d u}{\bar{F}_{2}(x) \int_{x}^{\infty} \bar{F}_{1}(u) e^{-u w} d u} \\
& =\frac{E\left[X_{2}(z)-x \mid X_{2}(z)>x\right]}{E\left[X_{1}(z)-x \mid X_{1}(z)>x\right]} .
\end{aligned}
$$

Because of (13), $\phi$ is increasing in $x \geq 0$. To discover the behavior of $\phi$ with respect to $w$, we establish the following function of $i$ and $w$. For fixed $x \geq 0$, set

$$
\rho(i, w)=\int_{x}^{\infty} \bar{F}_{i}(u) e^{-u w} d u,
$$

where $\bar{F}_{i}(u)$ is $\mathrm{TP}_{2}$ in $(i, u) \in\{1,2\} \times[x, \infty)$ since $X_{1} \leq_{h r} X_{2}$, and readily $e^{-u w}$ is $\mathrm{RR}_{2}$ in $(u, z) \in[x, \infty) \times[0, \infty)$. Therefore, on using Lemma $2.1, \rho$ is $\mathrm{RR}_{2}$ in $(i, w) \in\{1,2\} \times[0, \infty)$, which means that $\phi$ is decreasing in $w \geq 0$, for each fixed $x \geq 0$. Notice that $W$, for each fixed $x \geq 0$, is a non-negative rv with pdf

$$
h(w \mid x)=\frac{\left[\int_{x}^{\infty} \bar{F}_{1}(u) e^{-u w} d u\right] h(w)}{\int_{0}^{\infty}\left[\int_{x}^{\infty} \bar{F}_{1}(u) e^{-u w} d u\right] h(w) d w}, \quad w \geq 0 .
$$

It is easy to show that $h(w \mid x)$ is $\mathrm{RR}_{2}$ in $(w, x) \in[0, \infty) \times[0, \infty)$, which shows $W$ is stochastically decreasing in $x$. Now, Lemma 1.1(ii) completes the proof of the theorem.

Example 2.5 Let $X_{1}$ and $X_{2}$ have exponential distributions with means $1 / \theta_{1}$ and $1 / \theta_{2}$, respectively, such that $\theta_{1}>\theta_{2}$. Suppose now that $Z$ has density $h(z)=\left(z+\theta_{1}\right)(z+$ $\left.\theta_{2}\right) e^{-z \theta} / c\left(\theta, \theta_{1}, \theta_{2}\right)$ where $\theta>0$ and $c\left(\theta, \theta_{1}, \theta_{2}\right)$ is the normalizing constant. Evidently, $X_{1} \leq_{h r} X_{2}$ and also $X_{1}(z) \leq_{r l m r} X_{2}(z)$, for all $z \geq 0$. The assumptions of Theorem 2.10 hold. By some calculation, one gets $l_{2}^{*}(x) / l_{1}^{*}(x)=\left(1+\theta_{1}(x+\theta)\right) /\left(1+\theta_{2}(x+\theta)\right)$, which increases in $x$ as expected in Theorem 2.10 .

\subsection{Multivariate extension}

The aim of this subsection is to extend some results in Section 2 to the multivariate setting. To this end, we propose the notion of multivariate relative hazard rate and multivariate relative mean residual life orders. First, we recall the multivariate hazard rate and the multivariate weak hazard rate orders as defined in [11].

Definition 2.3 Let $\mathbf{X}=\left(X_{1}, \ldots, X_{n}\right)$ and $\mathbf{Y}=\left(Y_{1}, \ldots, Y_{n}\right)$ be two random vectors with respective survival functions $\bar{F}$ and $\bar{G}$ defined by $\bar{F}(\mathbf{x})=P(\mathbf{X}>\mathbf{x})$ and $\bar{G}(\mathbf{x})=P(\mathbf{Y}>\mathbf{x}), \mathbf{x} \in \mathbb{R}^{n}$. We say that $\mathbf{X}$ is smaller than $\mathbf{Y}$ in multivariate hazard rate order (denoted $\mathbf{X} \preceq_{m h r} \mathbf{Y}$ ) if

$$
\bar{F}(\mathbf{x}) \bar{G}(\mathbf{y}) \leq \bar{F}(\mathbf{x} \wedge \mathbf{y}) \bar{G}(\mathbf{x} \vee \mathbf{y}),
$$

for every $\mathbf{x}=\left(x_{1}, \ldots, x_{n}\right)$ and $\mathbf{y}=\left(y_{1}, \ldots, y_{n}\right)$ in $\mathbb{R}^{n}$ where $\mathbf{x} \wedge \mathbf{y}=\left[\min \left(x_{1}, y_{1}\right), \ldots, \min \left(x_{n}, y_{n}\right)\right]$ and $\mathbf{x} \vee \mathbf{y}=\left[\max \left(x_{1}, y_{1}\right), \ldots, \max \left(x_{n}, y_{n}\right)\right]$. Similarly, we say that $\mathbf{X}$ is smaller than $\mathbf{Y}$ in multivariate weak hazard rate order (denoted $\left.\mathbf{X} \preceq_{m w h r} \mathbf{Y}\right)$ if

$$
\frac{\bar{G}(\mathbf{x})}{\bar{F}(\mathbf{x})} \text { is increasing in } \mathbf{x} \in\left\{\mathbf{x} \in \mathbb{R}^{n}: \bar{F}(\mathbf{x})>0\right\} .
$$


It not hard to see that the order $\preceq_{m h r}$ is weaker that $\preceq_{m w h r}$, namely,

$$
\mathbf{X} \preceq_{m h r} \mathbf{Y} \Rightarrow \mathbf{X} \preceq_{m w h r} \mathbf{Y} .
$$

The multivariate hazard rate gradient of a random vector $\mathbf{X}=\left(X_{1}, \ldots, X_{n}\right)$ with a partial differentiable sf $\bar{F}$ is given by $\mathbf{r}_{\mathbf{X}}=\left(r_{\mathbf{X}}^{(1)}, \ldots, r_{\mathbf{X}}^{(n)}\right)$ with

$$
r_{\mathbf{X}}^{(i)}(\mathbf{x})=\frac{\partial_{i} \bar{F}(\mathbf{x})}{\bar{F}(\mathbf{x})}, \quad \mathbf{x} \in\left\{\mathbf{x} \in \mathbb{R}^{n}: \bar{F}(\mathbf{x})>0\right\}
$$

where $\partial_{i} \bar{F}(\mathbf{x})$ the partial derivative of $\bar{F}(\mathbf{x})$ with respect to $x_{i}$. Notice that the multivariate weak hazard rate order is characterized in terms of $r_{\mathbf{X}}^{(i)}, i=1, \ldots, n$, that is,

$$
\mathbf{X} \preceq_{m w h r} \mathbf{Y} \quad \Leftrightarrow \quad r_{\mathbf{X}}^{(i)}(\mathbf{x}) \geq r_{\mathbf{Y}}^{(i)}(\mathbf{x}), \quad i=1, \ldots, n
$$

Now, we introduce the multivariate relative hazard rate order.

Definition 2.4 Let $\mathbf{X}=\left(X_{1}, \ldots, X_{n}\right)$ and $\mathbf{Y}=\left(Y_{1}, \ldots, Y_{n}\right)$ be two random vectors. We say that $\mathbf{X}$ is smaller than $\mathbf{Y}$ in multivariate relative hazard rate order (denoted $\mathbf{X} \preceq_{m r l h r} \mathbf{Y}$ ), if, for every $i=1, \ldots, n$,

$$
\frac{r_{\mathbf{Y}}^{(i)}(\mathbf{x})}{r_{\mathbf{X}}^{(i)}(\mathbf{x})} \text { is decreasing in } \mathbf{x} \text {. }
$$

The following result shows that the multivariate relative hazard rate order is closed under marginalization as shown below.

Theorem 2.11 Let $\mathbf{X}=\left(X_{1}, \ldots, X_{n}\right)$ and $\mathbf{Y}=\left(Y_{1}, \ldots, Y_{n}\right)$ be two random vectors. Then

$$
\mathbf{X} \preceq_{m r l h r} \mathbf{Y} \quad \Rightarrow \quad X_{i} \preceq_{r l h r} Y_{i}, \quad i=1, \ldots, n .
$$

Proof The proof is directly obtained since $r_{X_{i}}\left(x_{i}\right)=r_{\mathrm{X}}^{(i)}\left(-\infty, \ldots,-\infty, x_{i},-\infty, \ldots,-\infty\right)$ and $r_{Y_{i}}\left(x_{i}\right)=r_{\mathbf{Y}}^{(i)}\left(-\infty, \ldots,-\infty, x_{i},-\infty, \ldots,-\infty\right)$.

Now, we will extend the results of Theorem 2.1 to the multivariate context.

Theorem 2.12 Let $\mathbf{X}=\left(X_{1}, \ldots, X_{n}\right)$ and $\mathbf{Y}=\left(Y_{1}, \ldots, Y_{n}\right)$ be positive random vectors with respective marginal density functions $f_{i}$ and $g_{i}, i=1, \ldots, n$.

(i) If for all $i=1, \ldots, n, \lim _{x \rightarrow 0} \frac{g_{i}(x)}{f_{i}(x)} \leq 1$ then $\mathbf{X} \preceq_{m r l h r} \mathbf{Y}$ implies $\mathbf{X} \preceq_{m w h r} \mathbf{Y}$.

(ii) If $0<\lim _{x_{i} \rightarrow \infty} \frac{\bar{G}(\mathbf{x})}{\bar{F}(\mathbf{x})}<\infty, i=1, \ldots, n$ then $\mathbf{X} \preceq_{m r l h r} \mathbf{Y}$ implies $\mathbf{Y} \preceq_{m w h r} \mathbf{X}$.

Proof To prove (i), assume that $\mathbf{X} \preceq_{m r l h r} \mathbf{Y}$, then $\frac{r_{\mathbf{Y}}^{(i)}(\mathbf{x})}{r_{\mathbf{X}}^{(i)}(\mathbf{x})}$ is decreasing for every $i=1, \ldots, n$. First, note that because $\lim _{\mathbf{x} \rightarrow \mathbf{0}} \bar{F}(\mathbf{x})=\lim _{\mathbf{x} \rightarrow \mathbf{0}} \bar{G}(\mathbf{x})=1$ and $\lim _{\mathbf{x} \rightarrow \mathbf{0}} \partial_{i} \bar{G}(\mathbf{x})=\lim _{x_{i} \rightarrow 0} g_{i}\left(x_{i}\right)$, $\lim _{\mathbf{x} \rightarrow \mathbf{0}} \partial_{i} \bar{F}(\mathbf{x})=\lim _{x_{i} \rightarrow 0} f_{i}\left(x_{i}\right)$. Thus

$$
\frac{r_{\mathbf{Y}}^{(i)}(\mathbf{x})}{r_{\mathbf{X}}^{(i)}(\mathbf{x})} \leq \lim _{\mathbf{x} \rightarrow \mathbf{0}} \frac{\partial_{i} \bar{G}(\mathbf{x})}{\partial_{i} \bar{F}(\mathbf{x})} \frac{\bar{F}(\mathbf{x})}{\bar{G}(\mathbf{x})}=\lim _{x_{i} \rightarrow 0} \frac{g_{i}\left(x_{i}\right)}{f_{i}\left(x_{i}\right)} \leq 1
$$


Consequently, $r_{\mathbf{Y}}^{(i)}(\mathbf{x}) \leq r_{\mathbf{X}}^{(i)}(\mathbf{x})$ for any $i=1, \ldots, n$, which ensure that $\mathbf{X} \preceq_{m w h r} \mathbf{Y}$. To show (ii), first observe from the L'Hospital rule

$$
\begin{aligned}
\lim _{x_{i} \rightarrow \infty} \frac{\partial_{i} \bar{G}(\mathbf{x})}{\partial_{i} \bar{F}(\mathbf{x})} & =\lim _{x_{i} \rightarrow \infty} \frac{\bar{G}(\mathbf{x})}{\bar{F}(\mathbf{x})} \\
& =\left[\lim _{x_{i} \rightarrow \infty} \frac{\bar{F}(\mathbf{x})}{\bar{G}(\mathbf{x})}\right]^{-1},
\end{aligned}
$$

because $\lim _{x_{i} \rightarrow \infty} \bar{F}(\mathbf{x})=\lim _{x_{i} \rightarrow \infty} \bar{G}(\mathbf{x})=0$. Therefore, $\mathbf{X} \preceq_{m r l h r} \mathbf{Y}$ implies

$$
\begin{aligned}
\frac{r_{\mathbf{Y}}^{(i)}(\mathbf{x})}{r_{\mathbf{X}}^{(i)}(\mathbf{x})} & \geq \lim _{x_{1} \rightarrow \infty} \frac{\partial_{i} \bar{G}(\mathbf{x})}{\partial_{i} \bar{F}(\mathbf{x})} \frac{\bar{F}(\mathbf{x})}{\bar{G}(\mathbf{x})} \\
& =\lim _{x_{i} \rightarrow \infty} \frac{\partial_{i} \bar{G}(\mathbf{x})}{\partial_{i} \bar{F}(\mathbf{x})} \lim _{x_{i} \rightarrow \infty} \frac{\bar{F}(\mathbf{x})}{\bar{G}(\mathbf{x})} \\
& \geq 1,
\end{aligned}
$$

and hence $\mathbf{Y} \preceq_{m w h r} \mathbf{X}$.

Now, we will provide the multivariate version of Theorem 2.2 .

Theorem 2.13 Let $\mathbf{X}=\left(X_{1}, \ldots, X_{n}\right)$ and $\mathbf{Y}=\left(Y_{1}, \ldots, Y_{n}\right)$ be non-negative random vectors such that for all $i=1, \ldots, n, \lim _{\mathbf{x} \rightarrow \infty} \frac{r_{\mathbf{Y}}^{(i)}(\mathbf{x})}{r_{\mathbf{X}}^{(i)}(\mathbf{x})}>0$. If $\mathbf{X} \preceq_{m r l h r} \mathbf{Y}$, then there exists $\alpha>0$ such that

$$
\bar{G}(\mathbf{x}) \leq \bar{F}^{\alpha}(\mathbf{x}) \text { for all } \mathbf{x}>\mathbf{0} \text {. }
$$

Proof For any $\mathbf{x}=\left(x_{1}, \ldots, x_{n}\right)$ such that $x_{i}>0, i=1, \ldots, n$, one has

$$
\begin{aligned}
& \int_{0}^{x_{n}} r_{\mathbf{Y}}^{(n)}\left(x_{1}, \ldots, x_{n-1}, u\right) d u=-\ln [\bar{G}(\mathbf{x})]+\ln \left[\bar{G}\left(x_{1}, \ldots, x_{n-1}, 0\right)\right], \\
& \int_{0}^{x_{n-1}} r_{\mathbf{Y}}^{(n-1)}\left(x_{1}, \ldots, x_{n-2}, u, 0\right) d u=-\ln \left[\bar{G}\left(x_{1}, \ldots, x_{n-1}, 0\right)\right]+\ln \left[\bar{G}\left(x_{1}, \ldots, x_{n-2}, 0,0\right)\right], \\
& \vdots \\
& \int_{0}^{x_{2}} r_{\mathbf{Y}}^{(2)}\left(x_{1}, u, 0, \ldots, 0\right) d u=-\ln \left[\bar{G}\left(x_{1}, x_{2}, 0, \ldots, 0\right)\right]+\ln \left[\bar{G}\left(x_{1}, \ldots, 0\right)\right], \\
& \int_{0}^{x_{1}} r_{\mathbf{Y}}^{(1)}(u, 0, \ldots, 0) d u=-\ln \left[\bar{G}\left(x_{1}, 0, \ldots, 0\right)\right]+\ln [\bar{G}(0, \ldots, 0)]
\end{aligned}
$$

and hence

$$
-\ln [\bar{G}(\mathbf{x})]=\sum_{i=1}^{n} \int_{0}^{x_{i}} r_{\mathbf{Y}}^{(i)}\left(x_{1}, \ldots, x_{i-1}, u, 0, \ldots, 0\right) d u
$$

Since $\mathbf{X} \preceq_{m r l h r} \mathbf{Y}$, the function

$$
c_{i}\left(x_{1}, \ldots, x_{i-1}, u, 0, \ldots, 0\right)=\frac{r_{\mathbf{Y}}^{(i)}\left(x_{1}, \ldots, x_{i-1}, u, 0, \ldots, 0\right)}{r_{\mathbf{X}}^{(i)}\left(x_{1}, \ldots, x_{i-1}, u, 0, \ldots, 0\right)}
$$


is decreasing for every $i=1, \ldots, n$. In particular, one has

$$
c_{i}\left(x_{1}, \ldots, x_{i-1}, u, 0, \ldots, 0\right) \geq \lim _{\mathbf{x} \rightarrow \infty} \frac{r_{\mathbf{Y}}^{(i)}(\mathbf{x})}{r_{\mathbf{X}}^{(i)}(\mathbf{x})}=\alpha_{i}>0
$$

Setting $\alpha=\min \left(\alpha_{1}, \ldots, \alpha_{n}\right)>0$, it follows that

$$
\begin{aligned}
-\ln [\bar{G}(\mathbf{x})] & =\sum_{i=1}^{n} \int_{0}^{x_{i}} c_{i}\left(x_{1}, \ldots, x_{i-1}, u, 0, \ldots, 0\right) r_{\mathbf{X}}^{(i)}\left(x_{1}, \ldots, x_{i-1}, u, 0, \ldots, 0\right) d u \\
& \geq \sum_{i=1}^{n} \alpha_{i} \int_{0}^{x_{i}} r_{\mathbf{X}}^{(i)}\left(x_{1}, \ldots, x_{i-1}, u, 0, \ldots, 0\right) d u \\
& \geq \alpha \sum_{i=1}^{n} \int_{0}^{x_{i}} r_{\mathbf{X}}^{(i)}\left(x_{1}, \ldots, x_{i-1}, u, 0, \ldots, 0\right) d u \\
& =-\alpha \ln [\bar{F}(\mathbf{x})] .
\end{aligned}
$$

Thus, $\ln [\bar{G}(\mathbf{x})] \leq \alpha \ln [\bar{F}(\mathbf{x})]$, that is, $\bar{G}(\mathbf{x}) \leq \bar{F}^{\alpha}(\mathbf{x})$ and hence the proof is completed.

In [23], the concept of the mrl has been extended to the multivariate case. As stressed in [24], the multivariate $\mathrm{mrl}$ is particularly useful in modeling and analyzing multivariate failure data when there is a lack of independence among the components (lifetimes). Such a situation might be due to outside conditions which affect all components. The concept of a multivariate mrl introduced in [23] is given hereafter.

Definition 2.5 Let $\mathbf{X}=\left(X_{1}, \ldots, X_{n}\right)$ be a random vectors with sf $\bar{F}$. The multivariate mrl function associated to $\mathbf{X}$ is defined for all $\mathbf{t} \in\left\{\mathbf{t} \in \mathbb{R}^{n}: \bar{F}(\mathbf{t})>0\right\}$ by

$$
m_{\mathbf{X}}(\mathbf{t})=\left[m^{(1)}(\mathbf{t}), \ldots, m^{(n)}(\mathbf{t})\right]
$$

where

$$
\begin{aligned}
m_{\mathbf{X}}^{(i)}(\mathbf{t}) & =E\left[X_{i}-t_{i} \mid \mathbf{X} \geq \mathbf{t}\right] \\
& =\frac{1}{\bar{F}(\mathbf{t})} \int_{t_{i}}^{\infty} \bar{F}\left(x_{(i)}, \mathbf{t}\right) d x, \quad i=1, \ldots, n,
\end{aligned}
$$

with $\left(x_{(i)}, \mathbf{t}\right)=\left(t_{1}, \ldots, t_{i-1}, x, t_{i}, \ldots, t_{n}\right)$.

Let $\mathbf{X}$ be a random vector with a partial differentiable sf $\bar{F}$. In [23], it is pointed out that the multivariate $\mathrm{mrl} m_{\mathbf{X}}(\mathbf{t})$ and the multivariate hr gradient $r_{\mathbf{X}}(\mathbf{t})$ are related by

$$
r_{\mathbf{X}}^{(i)}(\mathbf{t})=\frac{\partial_{i} m_{\mathbf{X}}^{(i)}(\mathbf{t})+1}{m_{\mathbf{X}}^{(i)}(\mathbf{t})} .
$$

Based on this definition, one can introduce the multivariate mrl order and the multivariate relative mean residual life order as announced below.

Definition 2.6 Let $\mathbf{X}=\left(X_{1}, \ldots, X_{n}\right)$ and $\mathbf{Y}=\left(Y_{1}, \ldots, Y_{n}\right)$ be two random vectors. We say that $\mathbf{X}$ is smaller than $\mathbf{Y}$ in multivariate mrl order, denoted by $\mathbf{X} \preceq_{m m r} \mathbf{Y}$, if $m_{\mathbf{X}}^{(i)}(\mathbf{t}) \leq m_{\mathbf{Y}}^{(i)}(\mathbf{t})$ 
for all $i=1, \ldots, n$. Similarly, we say that $\mathbf{X}$ is smaller than $\mathbf{Y}$ in multivariate relative mean residual life order, denoted by $\mathbf{X} \preceq_{m r l m r} \mathbf{Y}$, if for all $i=1, \ldots, n$,

$$
\frac{m_{\mathbf{Y}}^{(i)}(\mathbf{t})}{m_{\mathbf{X}}^{(i)}(\mathbf{t})} \text { increasing in } \mathbf{t} .
$$

These stochastic orders are closed under marginalization as stated next.

Lemma 2.2 Let $\mathbf{X}=\left(X_{1}, \ldots, X_{n}\right)$ and $\mathbf{Y}=\left(Y_{1}, \ldots, Y_{n}\right)$ be two random vectors, then

$$
\mathbf{X} \preceq_{m m r} \mathbf{Y} \quad \Rightarrow \quad X_{i} \preceq_{m r} Y_{i}, \quad i=1, \ldots, n,
$$

and

$$
\mathbf{X} \preceq_{m r l m r} \mathbf{Y} \quad \Rightarrow \quad X_{i} \preceq_{r l m r} Y_{i}, \quad i=1, \ldots, n .
$$

Proof The result is an immediate consequence of the fact that, for all $i=1, \ldots, n$,

$$
m_{X_{i}}\left(t_{i}\right)=m_{\mathbf{X}}^{(i)}\left(-\infty, \ldots,-\infty, t_{i},-\infty, \ldots,-\infty\right)
$$

and

$$
m_{Y_{i}}\left(t_{i}\right)=m_{\mathbf{Y}}^{(i)}\left(-\infty, \ldots,-\infty, t_{i},-\infty, \ldots,-\infty\right)
$$

where $m_{X_{i}}\left(t_{i}\right)$ and $m_{Y_{i}}\left(t_{i}\right)$ denote the mrl functions of $X_{i}$ and $Y_{i}$, respectively.

The next result generalizes the result of Theorem 2.3 to the multivariate case.

Theorem 2.14 Let $\mathbf{X}=\left(X_{1}, \ldots, X_{n}\right)$ and $\mathbf{Y}=\left(Y_{1}, \ldots, Y_{n}\right)$ be random vectors with respective survival functions $\bar{F}$ and $\bar{G}$. Denote by $f_{i}$ and $g_{i}, i=1, \ldots, n$, their marginal density functions, respectively.

(i) If $\mathbf{X} \preceq_{m r l m r} \mathbf{Y}$ and $\mathbf{X} \preceq_{m m r} \mathbf{Y}$ then $\mathbf{Y} \preceq_{m w h r} \mathbf{X}$.

(ii) If $0<\lim _{t_{i} \rightarrow \infty} \frac{\bar{G}(\mathbf{t})}{\bar{F}(\mathbf{t})}<\infty, i=1, \ldots, n$, then $\mathbf{X} \preceq_{m r l m r} \mathbf{Y}$ implies $\mathbf{X} \preceq_{m w h r} \mathbf{Y}$.

(iii) If $E\left(X_{i}\right) \leq E\left(Y_{i}\right), i=1, \ldots, n$, then $\mathbf{X} \preceq_{m r l m r} \mathbf{Y}$ implies $\mathbf{X} \preceq_{m m r} \mathbf{Y}$.

Proof To show (i), first observe that

$$
\frac{G(\mathbf{t})}{F(\mathbf{t})}=\frac{m_{\mathbf{X}}^{(i)}(\mathbf{t})}{m_{\mathbf{Y}}^{(i)}(\mathbf{t})} \frac{\int_{t_{i}}^{\infty} \bar{G}\left(x_{(i)}, \mathbf{t}\right) d x}{\int_{t_{i}}^{\infty} \bar{F}\left(x_{(i)}, \mathbf{t}\right) d x} .
$$

Now, $\mathbf{X} \preceq_{m r l m r} \mathbf{Y}$ and $\mathbf{Y} \preceq_{m w h r} \mathbf{X}$ imply that $\mathbf{t} \mapsto \frac{m_{\mathbf{X}}^{(i)}(\mathbf{t})}{m_{\mathbf{Y}}^{(i)}(\mathbf{t})}$ and $\mathbf{t} \mapsto \frac{\int_{t_{i}}^{\infty} \bar{G}\left(x_{(i)}, \mathbf{t}\right) d x}{\int_{t_{i}}^{\infty} \bar{F}\left(x_{(i)}, \mathbf{t}\right) d x}$, respectively, are non-negative decreasing functions. Therefore, $\mathbf{t} \mapsto G(\mathbf{t}) / F(\mathbf{t})$ is decreasing, which ensures that $\mathbf{Y} \preceq_{m w h r} \mathbf{X}$. To prove (ii), first assume that $0<\lim _{t_{i} \rightarrow \infty} \bar{G}(\mathbf{t}) / \bar{F}(\mathbf{t})<\infty, i=1, \ldots, n$. Then, from the L'Hospital rule, one has

$$
\lim _{t_{i} \rightarrow \infty} \frac{\int_{t_{i}}^{\infty} \bar{G}\left(x_{(i)}, \mathbf{t}\right) d x}{\int_{t_{i}}^{\infty} \bar{F}\left(x_{(i)}, \mathbf{t}\right) d x}=\lim _{t_{i} \rightarrow \infty} \bar{G}(\mathbf{t}) / \bar{F}(\mathbf{t})=\left[\lim _{t_{i} \rightarrow \infty} \bar{F}(\mathbf{t}) / \bar{G}(\mathbf{t})\right]^{-1} .
$$




\section{Consequently,}

$$
\begin{aligned}
\frac{m_{\mathbf{Y}}^{(i)}(\mathbf{t})}{m_{\mathbf{X}}^{(i)}(\mathbf{t})} & \leq \lim _{t_{i} \rightarrow \infty} \frac{\int_{t_{i}}^{\infty} \bar{G}\left(x_{(i)}, \mathbf{t}\right) d x}{\int_{t_{i}}^{\infty} \bar{F}\left(x_{(i)}, \mathbf{t}\right) d x} \lim _{t_{i} \rightarrow \infty} \bar{F}(\mathbf{t}) / \bar{G}(\mathbf{t}) \\
& =1
\end{aligned}
$$

Finally, if $E\left(X_{i}\right) \leq E\left(Y_{i}\right), i=1, \ldots, n$ and $\mathbf{X} \preceq_{m r l m r} \mathbf{Y}$ then

$$
\begin{aligned}
\frac{m_{\mathbf{Y}}^{(i)}(\mathbf{t})}{m_{\mathbf{X}}^{(i)}(\mathbf{t})} & \geq \frac{m_{\mathbf{Y}}^{(i)}(\mathbf{0})}{m_{\mathbf{X}}^{(i)}(\mathbf{0})} \\
& =\frac{E\left[Y_{i}\right]}{E\left[X_{i}\right]} \\
& \geq 1,
\end{aligned}
$$

which completes the proof of (iii).

Example 2.6 To illustrate the usefulness of Theorem 2.14, let us consider the survival function of the bivariate-Pareto family with scale parameters $\sigma_{1}>0$ and $\sigma_{2}>0$ and the shape parameter $\alpha>1$ given by

$$
\bar{F}_{\sigma_{1}, \sigma_{2}, \alpha}\left(x_{1}, x_{2}\right)=\left[1+\frac{x_{1}}{\sigma_{1}}+\frac{x_{2}}{\sigma_{2}}\right]^{-\alpha}, \quad x_{1} \geq 0, x_{2} \geq 0
$$

Let $m_{\sigma_{1}, \sigma_{2}, \alpha}\left(t_{1}, t_{2}\right)=\left[m_{\sigma_{1}, \sigma_{2}, \alpha}^{(1)}\left(t_{1}, t_{2}\right), m_{\sigma_{1}, \sigma_{2}, \alpha}^{(2)}\left(t_{1}, t_{2}\right)\right], t_{1}, t_{2} \geq 0$ be the bivariate mean residual life function associated to $\bar{F}_{\sigma_{1}, \sigma_{2}, \alpha}$. Carrying out easy integrations we get

$$
\begin{aligned}
m_{\sigma_{1}, \sigma_{2}, \alpha}^{(1)}\left(t_{1}, t_{2}\right) & =\frac{\int_{t_{1}}^{\infty} \bar{F}_{\sigma_{1}, \sigma_{2}, \alpha}\left(u, t_{2}\right) d u}{\bar{F}_{\sigma_{1}, \sigma_{2}, \alpha}\left(t_{1}, t_{2}\right)} \\
& =\frac{\sigma_{1}}{1-\alpha}\left[1+\frac{t_{1}}{\sigma_{1}}+\frac{t_{2}}{\sigma_{2}}\right], \quad t_{1}, t_{2} \geq 0,
\end{aligned}
$$

and

$$
\begin{aligned}
m_{\sigma_{1}, \sigma_{2}, \alpha}^{(2)}\left(t_{1}, t_{2}\right) & =\frac{\int_{t_{2}}^{\infty} \bar{F}_{\sigma_{1}, \sigma_{2}, \alpha}\left(t_{1}, u\right) d u}{\bar{F}_{\sigma_{1}, \sigma_{2}, \alpha}\left(t_{1}, t_{2}\right)} \\
& =\frac{\sigma_{2}}{1-\alpha}\left[1+\frac{t_{1}}{\sigma_{1}}+\frac{t_{2}}{\sigma_{2}}\right], \quad t_{1}, t_{2} \geq 0 .
\end{aligned}
$$

Let $\mathbf{X}_{1}=\left(X_{1}, X_{2}\right)$ and $\mathbf{Y}_{2}=\left(Y_{1}, Y_{2}\right)$ be a random pairs with mean residual life functions $m_{\sigma_{1}, \sigma_{2}, \alpha}\left(t_{1}, t_{2}\right)$ and $m_{\sigma_{1}^{\prime}, \sigma_{2}^{\prime}, \alpha^{\prime}}\left(t_{1}, t_{2}\right), t_{1}, t_{2} \geq 0$. In order to characterize the order $\preceq_{m r l m r}$ in terms of scale and shape parameters, first observe that

$$
\frac{m_{\sigma_{1}^{\prime}, \sigma_{2}^{\prime}, \alpha^{\prime}}^{(1)}\left(t_{1}, t_{2}\right)}{m_{\sigma_{1}, \sigma_{2}, \alpha}^{(1)}\left(t_{1}, t_{2}\right)} \propto \frac{m_{\sigma_{1}^{\prime}, \sigma_{2}^{\prime}, \alpha^{\prime}}^{(2)}\left(t_{1}, t_{2}\right)}{m_{\sigma_{1}, \sigma_{2}, \alpha}^{(2)}\left(t_{1}, t_{2}\right)} .
$$


Thus, one has

$$
\begin{aligned}
& \mathbf{X} \preceq_{m r m r} \mathbf{Y} \Leftrightarrow \frac{m_{\sigma_{1}^{\prime}, \sigma_{2}^{\prime}, \alpha^{\prime}}^{(1)}\left(t_{1}, t_{2}\right)}{m_{\sigma_{1}, \sigma_{2}, \alpha}^{(1)}\left(t_{1}, t_{2}\right)} \text { is increasing in } t_{1}, t_{2} \geq 0 \\
& \Leftrightarrow \phi\left(t_{1}, t_{2}\right)=\frac{\sigma_{2}^{\prime} t_{1}+\sigma_{1}^{\prime} t_{2}+\sigma_{1}^{\prime} \sigma_{2}^{\prime}}{\sigma_{2} t_{1}+\sigma_{1} t_{2}+\sigma_{1} \sigma_{2}} \text { is increasing in } t_{1}, t_{2} \geq 0 \\
& \Leftrightarrow \quad \frac{\partial \phi\left(t_{1}, t_{2}\right)}{\partial t_{1}} \geq 0 \quad \text { and } \quad \frac{\partial \phi\left(t_{1}, t_{2}\right)}{\partial t_{2}} \geq 0 \quad \text { for all } t_{1}, t_{2} \geq 0 \\
& \Leftrightarrow \begin{cases}\sigma_{2}^{\prime}\left(\sigma_{1} \sigma_{2}-\sigma_{1}^{\prime} \sigma_{2}\right)+\left(\sigma_{1} \sigma_{2}^{\prime}-\sigma_{1}^{\prime} \sigma_{2}\right) t_{2} \geq 0 & \text { for all } t_{2} \geq 0, \\
\sigma_{1}\left(\sigma_{1}^{\prime} \sigma_{2}-\sigma_{1}^{\prime} \sigma_{2}^{\prime}\right)+\left(\sigma_{1}^{\prime} \sigma_{2}-\sigma_{1} \sigma_{2}^{\prime}\right) t_{1} \geq 0 & \text { for all } t_{1} \geq 0\end{cases} \\
& \Leftrightarrow \begin{cases}\sigma_{2}^{\prime}\left(\sigma_{1} \sigma_{2}-\sigma_{1}^{\prime} \sigma_{2}\right)+\left(\sigma_{1} \sigma_{2}^{\prime}-\sigma_{1}^{\prime} \sigma_{2}\right) t_{2} \geq 0 & \text { for all } t_{2} \geq 0, \\
\sigma_{1}\left(\sigma_{1}^{\prime} \sigma_{2}-\sigma_{1}^{\prime} \sigma_{2}^{\prime}\right)+\left(\sigma_{1}^{\prime} \sigma_{2}-\sigma_{1} \sigma_{2}^{\prime}\right) t_{1} \geq 0 & \text { for all } t_{1} \geq 0\end{cases} \\
& \Leftrightarrow \quad \frac{\sigma_{1}^{\prime}}{\sigma_{1}}=\frac{\sigma_{2}^{\prime}}{\sigma_{2}} \leq 1 \text {. }
\end{aligned}
$$

Now, we use Theorem 2.14 to establish a sufficient condition for the order $\preceq_{m w h r}$. Clearly,

$$
0<\lim _{x_{i} \rightarrow \infty} \frac{\bar{F}_{\sigma_{1}^{\prime}, \sigma_{2}^{\prime}, \alpha^{\prime}}\left(x_{1}, x_{2}\right)}{\bar{F}_{\sigma_{1}, \sigma_{2}, \alpha}\left(x_{1}, x_{2}\right)}<\infty, \quad i=1,2 \quad \Leftrightarrow \quad \alpha=\alpha^{\prime} .
$$

Thus, from (ii) of Theorem 2.14, one has

$$
\alpha=\alpha^{\prime} \quad \text { and } \quad \frac{\sigma_{1}^{\prime}}{\sigma_{1}}=\frac{\sigma_{2}^{\prime}}{\sigma_{2}} \leq 1 \Rightarrow \mathbf{X} \preceq_{m w h r} \mathbf{Y}
$$

We use again Theorem 2.14 to obtain a sufficient condition for the order $\preceq_{m w h r}$. Indeed, one can easily derive the next equivalence

$$
E\left(X_{i}\right) \leq E\left(Y_{i}\right), \quad i=1,2 \quad \Leftrightarrow \quad \frac{\sigma_{1}}{1-\alpha} \leq \frac{\sigma_{1}^{\prime}}{1-\alpha^{\prime}} \text { and } \frac{\sigma_{2}}{1-\alpha} \leq \frac{\sigma_{2}^{\prime}}{1-\alpha^{\prime}} .
$$

It follows that

$$
\begin{aligned}
& \mathbf{X} \preceq_{m r l m r} \mathbf{Y} \quad \text { and } \quad E\left(X_{i}\right) \leq E\left(Y_{i}\right), \quad i=1,2 \quad \Leftrightarrow \quad \alpha \leq \alpha^{\prime}, \\
& \mathbf{X} \preceq_{m r l m r} \mathbf{Y} \quad \text { and } \quad E\left(X_{i}\right) \leq E\left(Y_{i}\right), \quad i=1,2 \quad \Leftrightarrow \quad \alpha \leq \alpha^{\prime} \text { and } \frac{\sigma_{1}^{\prime}}{\sigma_{1}}=\frac{\sigma_{2}^{\prime}}{\sigma_{2}} \leq 1 .
\end{aligned}
$$

From (iii) of Theorem 2.14, we get

$$
\alpha \leq \alpha^{\prime} \quad \text { and } \quad \frac{\sigma_{1}^{\prime}}{\sigma_{1}}=\frac{\sigma_{2}^{\prime}}{\sigma_{2}} \leq 1 \quad \Rightarrow \quad \mathbf{X} \preceq_{m m r} \mathbf{Y}
$$

In addition, we observe from this analysis that the order $\preceq_{r m r m}$ is only affected by the scale parameters.

\section{Conclusion}

Most of the stochastic orders discovered and analyzed in the literature are the ones which compare the 'location' or the 'magnitude' of the random variables and there are other ones 
which compare the 'variability' or the 'dispersion' of the random variables ( $c f$. [11] and [12]). In this paper, as another perspective of stochastic comparison between lifetime variables, we devoted our attention to other kinds of stochastic orders which compare the random variables with respect to their 'aging' properties according to the well-known reliability measures of hazard rate and mean residual life. These stochastic orders, called the relative hazard rate order and the relative mean residual life order, have been somewhat neglected in the literature. We provide the interested reader with further properties of these stochastic orders in both the univariate and the multivariate cases. On the other hand, because there is no theoretical base for choosing the distribution of the additive effect variable in the additive frailty model, it is important to see how the overall variable is influenced by the variation of the additive effect and baseline variables in the model. Such an investigation has been recently done in the context of various reliability models (see, for example, [15] and the references therein). Thus, as a second lead, we use the relative hazard and the relative mean residual life orders for comparison of additive hazards model and we discussed various aspects of the study. Possible extensions to the multivariate case are discussed.

\section{Competing interests}

The authors declare that there is no conflict of interests regarding the publication of this paper.

\section{Authors' contributions}

All of the authors have equally made contributions. All authors read and approved the final manuscript.

\section{Author details}

${ }^{1}$ Permanent address: Department of Mathematics and Computer Science, Faculty of Science, Suez University, Suez, 41522, Egypt. ${ }^{2}$ Department of Statistics and Operations Research, College of Science, King Saud University, Riyadh, Saudi Arabia. ${ }^{3}$ Department of Statistics, Faculty of Mathematical Sciences, Ferdowsi University of Mashhad, Mashhad, Iran. ${ }^{4}$ Université du Québec à Trois-Rivières, C.P. 500, Québec, G9A 5H7, Canada.

\section{Acknowledgements}

The authors want to thank two anonymous referees for their constructive comments decisively contributed to improve the paper. The authors would like to extend their sincere appreciation to the Deanship of Scientific Research at King Saud University for funding this Research Group No. (RGP-1435-036). The third author thanks the Natural Sciences and Engineering Research Council of Canada.

Received: 29 December 2015 Accepted: 8 June 2016 Published online: 17 June 2016

\section{References}

1. Di Crescenzo, A: Some results on the proportional reversed hazards model. Stat. Probab. Lett. 50, 313-321 (2000)

2. Badia, FG, Berrade, MD, Campos, CA: Aging properties of the additive and proportional hazard mixing models. Reliab. Eng. Syst. Saf. 78, 165-172 (2002)

3. Sankaran, PG, Jayakumar, K: On proportional odds models. Stat. Pap. 49, 779-789 (2008)

4. Oliveira, PE, Torrado, N: On proportional reversed failure rate class. Stat. Pap. (2014). doi:10.1007/s00362-014-0620-8

5. Das, S, Nanda, AK: Some stochastic orders of dynamic additive mean residual life model. J. Stat. Plan. Inference 143, 400-407 (2013)

6. Nanda, AK, Das, S, Balakrishnan, N: On dynamic proportional mean residual life model. Probab. Eng. Inf. Sci. 27, 553-588 (2013)

7. Hanagal, DD: Modeling heterogeneity for bivariate survival data by the Weibull distribution. Stat. Pap. 51, 947-958 (2010)

8. Nair, NU, Sankaran, PG: Some results on an additive hazards model. Metrika 75, 389-402 (2012)

9. Gupta, RC, Gupta, RD: General frailty model and stochastic orderings. J. Stat. Plan. Inference 139, 3277-3287 (2009)

10. Nanda, AK, Bhattacharjee, S, Alam, SS: Properties of aging intensity function. Stat. Probab. Lett. 77, 365-373 (2007)

11. Shaked, M, Shanthikumar, JG: Stochastic Orders. Springer, New York (2007)

12. Müller, A, Stoyan, D: Comparison Methods for Stochastic Models and Risks. Wiley, New York (2002)

13. Karlin, S: Total Positivity. Stanford University Press, Stanford (1968)

14. Misra, N, Van der Meulen, EC: On stochastic properties of $m$-spacings. J. Stat. Plan. Inference 115, 683-697 (2003)

15. Gupta, RC, Peng, C: Proportional odds frailty model and stochastic comparisons. Ann. Inst. Stat. Math. 66, 897-912 (2014)

16. Sengupta, D, Deshpande, JV: Some results on the relative ageing of two life distributions. J. Appl. Probab. 31, 991-1003 (1994)

17. Kayid, M, Izadkhah, S, Zuo, MJ: Some results on the relative ordering of two frailty models. Stat. Pap. (2015). doi:10.1007/s00362-015-0697-8 
18. Hu, T, Kundu, A, Nanda, AK: On generalized orderings and ageing properties with their implications. In: Hayakawa, Y, Irony, T, Xie, M (eds.) System and Bayesian Reliability: Essays in Honor of Prof. R.E. Barlow, pp. 199-228. World Scientific, Hackensack (2001)

19. Pocock, SJ, Gore, SM, Kerr, GR: Long-term survival analysis: the curability of breast cancer. Stat. Med. 1, 93-104 (1982)

20. Wei, X: Relative mean residual life: theory and related topics. Microelectron. Reliab. 32, 1319-1326 (1992)

21. Finkelstein, M: On relative ordering of mean residual lifetime functions. Stat. Probab. Lett. 76, 939-944 (2006)

22. Nanda, AK, Bhattacharjee, S, Balakrishnan, N: Mean residual life function, associated orderings and properties. IEEE Trans. Reliab. 59, 55-65 (2010)

23. Arnold, BC, Zahedi, H: On multivariate mean remaining life functions. J. Multivar. Anal. 25, 1-9 (1988)

24. Zahedi, H: Some new classes of multivariate survival distribution functions. J. Stat. Plan. Inference 11, 171-188 (1985)

\section{Submit your manuscript to a SpringerOpen ${ }^{\circ}$ journal and benefit from:}

- Convenient online submission

Rigorous peer review

- Immediate publication on acceptance

- Open access: articles freely available online

- High visibility within the field

- Retaining the copyright to your article 